\title{
Correction to: A placebo-controlled, double-blind, randomized study of recombinant thrombomodulin (ART-123) to prevent oxaliplatin-induced peripheral neuropathy
}

\author{
Masahito Kotaka' ${ }^{1}$ (1) Yoji Saito ${ }^{2} \cdot$ Takeshi Kato $^{3,4} \cdot$ Hironaga Satake $^{5,6} \cdot$ Akitaka Makiyama $^{7,8} \cdot$ Yasushi Tsuji $^{9}$. \\ Katsunori Shinozaki ${ }^{10}$. Toshiyoshi Fujiwara ${ }^{11}$. Tsunekazu Mizushima ${ }^{12}$. Yasushi Harihara ${ }^{13}$. Naoki Nagata ${ }^{14}$. \\ Naoto Kurihara ${ }^{15}$. Masahiko Ando ${ }^{16}$. Genichi Kusakawa ${ }^{17} \cdot$ Takumi Sakai $^{17} \cdot$ Yugo Uchida ${ }^{17} \cdot$ Mikihiro Takamoto $^{17}$. \\ Saki Kimoto ${ }^{17} \cdot$ Ichinosuke Hyodo ${ }^{18,19}$
}

Published online: 2 November 2020

(c) Springer-Verlag GmbH Germany, part of Springer Nature 2020

\section{Correction to: \\ Cancer Chemotherapy and Pharmacology \\ (2020) 86:607-618 \\ https://doi.org/10.1007/s00280-020-04135-8}

In the Original publication of the article, the authors found an error in the "Results" section under the heading "Abstract". The corrected text is given below:

The original article can be found online at https://doi.org/10.1007/ s00280-020-04135-8

Masahito Kotaka

tomomakotaka6410@yahoo.co.jp

1 Gastrointestinal Cancer Center, Sano Hospital, 2-5-1 Shimizugaoka, Tarumi-ku, Kobe-shi, Hyogo 655-0031, Japan

2 Department of Anesthesiology, Shimane University Faculty of Medicine, 89-1 Enyacho, Izumo City, Shimane 693-8501, Japan

3 Department of Gastroenterological Surgery, Kansai Rosai Hospital, 3-1-69 Inabaso, Amagasaki-shi, Hyogo 660-8511, Japan

4 Colorectal Surgery, National Hospital Organization Osaka National Hospital, 2-1-14 Hoenzaka, Chuo-ku, Osaka City, Osaka 540-0006, Japan

5 Department of Medical Oncology, Kobe City Medical Center General Hospital, 2-1-1 Minatojimaminamimachi, Chuo-ku, Kobe-city, Hyogo 650-0047, Japan

6 Cancer Treatment Center, Kansai Medical University Hospital, 2-3-1 Shin-machi, Hirakata City, Osaka 573-1191, Japan
Seventy-nine participants (placebo $n=28,1$-day ART $n=27,3$-day ART $n=24$ ) received study drugs. The leastsquares mean FACT/GOG-Ntx-12 scores at cycle 12 from the mixed effect model for repeated measures were 28.9 with placebo, 36.3 with 1-day ART [vs. placebo: 7.3 (95\% CI 1.9 to $12.8, p=0.009)$ ], and 32.3 with 3 -day ART [vs. placebo: $3.4(95 \% \mathrm{CI}-2.1$ to $9.0, p=0.222)]$.
7 Department of Hematology/Oncology, Japan Community Healthcare Organization Kyushu Hospital, 1-8-1 Kishinoura, Yahatanishi-ku, Kitakyusyu-shi, Fukuoka 806-8501, Japan

8 Cancer Center, Gifu University Hospital, 1-1 Yanagido, Gifu 501-1194, Japan

9 Department of Medical Oncology, Tonan Hospital, Kita 4-jo Nishi 7-chome 3-8, Chuo-ku, Sapporo-shi, Hokkaido 060-0004, Japan

10 Division of Clinical Oncology, Hiroshima Prefectural Hospital, 1-5-54 Ujinakanda, Minami-ku, Hiroshima-shi, Hiroshima 734-8530, Japan

11 Department of Gastroenterological Surgery, Okayama University Graduate School of Medicine, Dentistry and Pharmaceutical Sciences, 2-5-1 Shikata-cho, Kita-ku, Okayama-city, Okayama 700-8558, Japan

12 Department of Gastrointestinal Surgery, Osaka University Graduate School of Medicine, 2-2 Yamadaoka, Suita, Osaka 565-0871, Japan

13 Department of Surgery, NTT Medical Center Tokyo, 5-9-22 Higashi-Gotanda, Shinagawa-ku, Tokyo 141-8625, Japan 
14 Department of Surgery, Kitakyushu General Hospital, 1-1 Higashijono-machi, Kokurakita-ku, Kitakyushu 802-8517, Japan

15 Department of Surgery, Nerima General Hospital, 1-24-1 Asahigaoka, Nerima-ku, Tokyo 176-8530, Japan

16 Center for Advanced Medicine and Clinical Research, Nagoya University Hospital, 65 Tsurumai-cho, Showa-ku, Nagoya 466-8560, Japan

17 Clinical Development Center, Asahi Kasei Pharma Corporation, 1-1-2 Yurakucho, Chiyoda-ku, Tokyo 100-0006, Japan
18 Division of Gastroenterology, University of Tsukuba, 1-1-1 Tennodai, Tsukuba, Ibaraki 305-8577, Japan

19 Division of Gastrointestinal Medical Oncology, NHO Shikoku Cancer Center, 160 Kou Minami-umemoto, Matsuyama-city, Ehime 791-0280, Japan

Publisher's Note Springer Nature remains neutral with regard to jurisdictional claims in published maps and institutional affiliations. 